\title{
Impact of Health Information Technologies in Nursing Practices
}

\author{
*Hilal UYSAL, \\ Assistant Professor, PhD,RN. \\ *Kübra Yeni, \\ Research Assistant, MSc.N. \\ *Istanbul University Florence Nightingale Faculty of Nursing, Medical Nursing Department, \\ Istanbul, Turkey.
}

Correspondence Writer: HiLAL UYSAL, Assistant Professor, PhD, RN.

\begin{abstract}
Telehealth implementation includes the practices that help giving care to individuals by monitoring them from a distance, generally in their homes, without making them come to the clinic. Telehealth services ensure that patients participate in their own care effectively, increase their self-direction to cope with the disease, and cover certain initiatives that reduce health-related costs, such as training and consultancy. With a fast development of technology, the use of technological appliances in the area of health would facilitate access to health services. Telehealth practices ensure that the persons who have difficulty accessing health services due to geograpghical and other reasons are provided with equal level of care. Moreover, they facilitate monitoring individuals with chronic diseases and increase the quality of care by providing communication and consultancy for those who give care in areas with technological insufficiencies. In this regard, fast-developing technological infrastructure and use of information technologies in nursing, as stressed by American Nursing Association, are very important today. This study suggested that the primary goals in the future should be to increase the costeffectiveness of health care and strengthen the competencies of professionals and institutions by implementing proper health policies.
\end{abstract}

Keywords: Telenursing, telehealth, telecare, nursing.

\section{INTRODUCTION}

Chronic illnesses have become more common with the development of new treatment methods and extension of lifespan. This has turned into a burden for individuals and national economy. For this reason, the use of technology in health care is essential for reducing expenses on health. The provision of telehealth and telehealth nursing services have become important both in Turkey and in the world in recent years. Telehealth is the use of communication technologies in the delivery of health care services [1].

The areas of telehealth are home health care (monitoring, education, support), nursing home (specialty consultations), ambulatory care (specialty consultations), hospital consultations (stroke care), prison settings (mental health services), mentoring (i.e., wound care specialists), intensive care unit (ICU) monitoring (neonatal intensive care, cardiac care), and health promotion (obesity, exercise/fitness). Telehealth implementation includes the practices that help giving care to individuals by monitoring them from a distance, generally in their homes, without making them come to the clinic. Telehealth services ensure that patients participate in their own care effectively, increase their self-direction to cope with the disease, and cover certain initiatives that reduce health-related costs, such as training and consultancy [2-4]. Telehealth practices were included in the literature in the 1970s, yet they were not defined clearly. These practices were intended to provide clinical support, ensure that the individuals who have difficulty accessing health care and geographical limitations gain equal rights to health care, and improve health care results by using different methods of communication in the health area [5].

The most fundamental benefits of using information technologies in nursing care are having a record of individuals' care and treatment, monitoring the changes in patients' status, following the health care 
results statistically, and evaluating and sharing the results with other health care professionals. It also provides fast access to patients' records, which facilitates the determination of patients' care needs and the provision of suitable care. Thanks to the information technologies and recording systems, all information about patients and the results of all initiatives from their admission to discharge are easily accessed now. These practices also make it possible to spend more time with patients. Despite few examples in this area, progress has been reported [6].

\section{BENEFITS AND Limitations OF TELEHEALTH APPLiCATIONS}

Video conference is one of the telehealth implementations, and it has been applied effectively in recent years. It makes it possible for health researchers from different countries to meet and share information. This implementation has been reported to be cost-effective [7]. A socioeconomic evaluation of the effectiveness of telehealth implementations, which are performed using several technological methods, showed that they facilitated the access to health implementations, improved health-related training opportunities and quality of life, and provided social support to patients and caregivers [8]. The benefits of telehealth include health care access, financial return, coordination of care, and quality of care.

Although telehealth implementations have yielded positive returns in many areas, their usage has certain limitations. Studies have reported that the barriers ahead of the common utilization of telehealth implementations are the unclear points regarding the sustainability of the continuation of telehealth implementations, expansion of usage, financial investment, technology infrastructure/interoperability, licensure and credentialing, reimbursement, evaluation/outcomes, insufficient policies to encourage telehealth implementations, and general lack of knowledge about telehealth implementations [9]. Moreover, ethical problems have been reported in telehealth practices. Most important ones of these ethical problems are the failure of protecting personal information, violation of patients' privacy, lack of communication between physicians and patients, and insufficient conveyance of information $[6,10]$.

Despite the limitations, 700,000 patients were provided with telehealth service in the United States in the year 2009. Considering the pace of getting old and facing chronic health problems in Europe, the use of telehealth services is gaining more importance every day. The Bosch Telehealth System, which is used in Europe, includes implementations that help in medical diagnosis and treatment such as blood pressure, blood glucose level, and oxygen saturation, as well as provide data about patients' health behaviors. Accordingly, the patients are evaluated extensively and provided with distance education and consultancy, which support their effective participation in care. It is emphasized that many evidence-based studies used this method. These studies were particularly conducted with elderly patients who had chronic obstructive pulmonary disease, diabetes, heart failure, and asthma. Moreover, telehealth implementations were effective, and many positive results were derived from these studies $[4,11]$.

In Turkey, an action plan was developed and put into force by the Ministry of Health in 006. One of the decisions about e-health made in this project was the realization of telemedicine systems. It was also decided that the action plan would be initiated for projects including central hospital appointment system, e-prescription, electronic referral system, family physicians information system, and electronic id card. The first stage of the project was activated in December 2007. The goals of the telemedicine project were to help eliminate the lack of screening specialists, improve patient satisfaction, minimize the loss of cost, time, and labor, implement accurate diagnosis and treatment operations, and ensure information-sharing among physicians [12].

\section{EvidenCe-BASED Practice IMPlications}

With a fast development of technology, the use of technological appliances in the area of health would facilitate access to health services. Telehealth practices ensure that the persons who have difficulty accessing health services due to geograpghical and other reasons are provided with equal level of care. Moreover, they facilitate monitoring individuals with chronic diseases and increase the quality of care by providing communication and consultancy for those who give care in areas with technological insufficiencies [5].

According to a systematic review about the telehealth services, which include video, web-based implementations, and remote monitoring, more than $95 \%$ of the studies indicated positive 
developments in caregiver results; they were satisfied with telehealth implementations and found it more accessible and easy to use. The review also found that telehealth implementations made important contributions to the care of patients with chronic diseases, home care, and hospice care [13]. A systematic review about the effectiveness of tele-monitoring in individuals with heart disease evaluated 18 studies and determined that this implementation produced positive results. It was also found that tele-monitoring reduced the hospital stay for patients with heart disease, helped determine the possible problems at an early stage, and reduced hospital costs. However, the review also reported that although tele-monitoring implementation was effective in care, further randomized, controlled studies were needed [14]. A study conducted with tetraplegic patients showed less deterioration in oral hygiene with the telecare method in short (6 months) and long term (12 months) and fewer gingival problems [15].

In recent years, it has been shown that telecare practices are effective in type 2 diabetes [16-20]. It was also found that diabetic patients had better glycemic control with tele-monitoring [16], and their hemoglobin A1c and postprandial glucose levels decreased [17-19]. Telehealth practices increased the adaptation for not only diabetes but also other chronic diseases [21]. Another study proved that [22] the computerized indicators used for nursing initiatives were beneficial for taking precautions against hypothermia, skin lesions, catheter-related problems, and falls that might happen during health care practices. These computer-aided tools made it possible to collect data faster.

\section{Historical Development of Telehealth and Telehealth Nursing in Turkey AND IN THE WORLD}

Telehealth practices began to be discussed in the 1950s in the world and in the 2000s in Turkey. In 2006, the Ministry of Health prepared the first action plan on this issue. In 2007, they established the tele-radiology, tele-pathology, and tele-electrocardiogram services. As a part of telehealth implementations, house care service began in 2010 [23].

In the world, the first article on telehealth nursing was written by Mary Quinn in 1974, who also provided hospital-centered telehealth nursing [24, 25]. The International Council of Nurses described telenursing in 2001 and reported that a sufficient number of equipped nurses were needed for the use of technology, as well as terminology and classification system. To ensure progress in the telehealth nursing field, two important projects (NIGHTINGALE Project and TeleNurse-ID) were launched in Europe (26). The overall objective of TeleNurse-ID (Project date 1991-2000) was to promote the use of the International Classification for Nursing Practice in Europe. Based on the results of both the Telenurse and TeleNurse-ID projects, nursing record components were matched with the existing clinical object dictionary. This activity was performed for various nursing record components: nursing assessment, nursing care process, nursing care plan, medical orders processing, vital signs chart, and nursing minimal data set abstracting [26]

The main objective of the NIGHTINGALE project was to develop nursing informatics curriculum, investigate the interest to include nursing informatics education in the nursing curriculum in nursing schools, and find out the informatics products in which nursing schools were interested through the dissemination of a questionnaire [27].

However, no international classification system exists in Turkey yet; the number of nurses equipped to use the current technology is insufficient. The limitations of institutions and the society in terms of technological equipment are the barriers ahead of the telehealth nursing practices [28].

\section{Telehealth NurSing}

Telenursing is the delivery, management, and coordination of care and services provided via telecommunications technology within the domain of nursing [29]. Telehealth nursing is practiced in the home, health care clinic, doctor's office, prisons, hospitals, telehealth nursing call centers, and mobile units. Telehealth nursing uses telecommunications technology (i.e., electromagnetic channels including wires, radios, and optics) to enhance patient care. Telephone triage, telecare remote monitoring, and home care are the fastest growing applications [24, 25]. Tele-home care helps to deliver nursing services in a faster and more economical way by providing necessary equipment to perform patients' physiological assessments from a distance, advising patients based on the determined problems, and providing them with the required care. In telephone triage implementations, this system ensures that individuals are directed to the relevant resources regarding their health 
problems. Telecare-remote monitoring implementations help collect and interpret patients' data, and direct them to the relevant health resources. In addition, these implementations offer social care services, which help individuals become more independent in their lives and perform self-care. Telehealth nursing implementations ensure that continuous and quality care is provided, patient satisfaction is increased, costs are reduced, performance is evaluated, and documentation and clinical guide are created [1,25].

The role of telehealth nursing is that of the nurse presenter (i.e., planning telehealth program, coordinating and scheduling patients, evaluating and preparing equipment, assessing patients, presenting patients, facilitating remote exam, documentation), nurse case manager (i.e., diabetes care), public health nursing, health coach, tele-ICU nursing, clinical telehealth coordinator, patient education, teleconsultations, conducting medical test/results, physicals exams/assessment, and collaboration with health care providers in implementing medical treatment protocols and providing follow-up care [25].

The importance and necessity of telehealth nursing implementations are emphasized by the fact that technological developments in the field of home care services are rapid, and individuals have a desire to live a long and quality life. With an integrated use of telecommunication network and health technologies, telehealth nursing makes it possible to improve patient care services. It also prevents repeated hospitalization and applications, provides cost-effectiveness, facilitates the managements of chronic diseases, and provides an increased access to nursing care and implementations. Other advantages of telehealth nursing are early determination and management of the symptoms of patients' diseases, increased patient-caregiver satisfaction and occupational management of nurses, reduction in sudden and unexpected situations thanks to continuous monitoring, and helping patients participate actively in their own care [24].

From this perspective, telehealth nursing is used in the management of many illnesses. For instance, patients diagnosed with cancer need a multidimensional care and monitoring starting from the diagnosis. They have to deal with many issues including the disease itself, the medication they use and their side effects, coping with the symptoms, and re-adaptation to social life. The involvement of telehealth nursing implementations help providing consultancy to patients, and the patients who have their diseases managed successfully maintain a healthy life [30]. A randomized controlled study examined the effectiveness of telecare implementations in the management of pain and depression in cancer patients. The study reported an increase in the quality of life of patients who were supported by telecare implementation for pain and depression, besides a significant reduction in expenses [31].

In diabetes, which is another chronic disease, nurses play a key role in planning, implementation, and evaluation of the training [30]. A study conducted by nurses reported that metabolic control was better and cardiovascular risk reduced when patients were monitored by telephone [18]. In recent years, a different study conducted with diabetic patients stated that the telehealth nursing implementation was successful in optimal glycemic control and patients' cognitive-social adaptation [20]. Telehealth practices performed with schizophrenic patients produced positive results including reduced hospitalization and increased adaptation to medication treatment [32]. Similar studies conducted in Turkey proved that patients who opted for tele-consultancy had a marked reduction in their care burden(unpublished thesis), an increase in their self-care (unpublished thesis), a considerable improvement in their adaptation to the changes in their lifestyle (unpublished thesis), reduced risky behaviors, and enhanced quality of life [33]. Telehealth nursing yields positive returns not only in chronic cases but also in emergency situations. When patients call the centers that provide 24 -h teleconsultancy, $40 \%$ of these patients are given accurate advice without the need to go to the center [1, $34]$. Further studies are needed to evaluate the effectiveness of telehealth nursing practices $[17,32]$.

Health professionals play an important role in the use of information technologies. Thus, it is necessary for nurses to be introduced to and trained on information technologies through undergraduate programs. Contents included in undergraduate and graduate programs, such as information technologies and nursing, will prepare nurses for professional life. Therefore, nurses must meet standards of care for safe, quality, and competent practice for telehealth nursing by practicing within the American Nurses Association (ANA) scope and standards of nursing practice. Specific telehealth standards can be found with the American Academy of Ambulatory Care Nurses and ANA [25]. ANA [35] has accepted nursing informatics as a specialty. Similar to many other countries in the world, Turkey also has initiated undergraduate and graduate programs. In addition, conferences and 
certificate programs are also organized on this subject. A study conducted in 2006 emphasized that the information given about using computer and nursing informatics was not sufficient in basic nursing training [6]. Today, undergraduate nursing students are trained on basic information technologies, computer usage, and nursing informatics, which shows that the importance of this subject has been realized and understood.

\section{Conclusions}

The most fundamental benefits of using information technologies in nursing care are having a record of individuals' care and treatment, monitoring the changes in patients' status, following the health care results statistically, and evaluating and sharing the results with other health care professionals. Therefore, this study suggested that the primary goals in the future should be to increase the costeffectiveness of health care and strengthen the competencies of professionals and institutions by implementing proper health policies.

\section{REFERENCES}

[1] Pazar B., Taştan S., İyigün E. Roles of nurses in tele-health services. Medical Journal of Bakirkoy, 11(1). (2015).

[2] Parker SG., Hawley MS. Telecare for an ageing population? Age and Ageing, 42(4),pp.424-5. (2013).

[3] Dixon BE., Hook JM., McGowan JJ. Using telehealth to improve quality and safety: Findings from the AHRQ Health IT portfolio: Agency for Healthcare Research and Quality. (2008)

[4] Klapper B., Kühne H. Patient self-management by telehealth using the Bosch model of care. Journal of Telemedicine and Telecare, 16(4),pp.193-5. (2010).

[5] Telemedicine W (2009). Opportunities and Developments in Member States: Report on the Second Global Survey on eHealth; 2,pp.37-49. (2009).

[6] Bilgiç Ş., Şendir M. Nursing Informatics. Cumhuriyet Nursing Journal, 3(1),pp.24-8. (2014).

[7] Boatin A., Ngonzi J., Bradford L., Wylie B., Goodman A. Teaching by Teleconference: A Model for Distance Medical Education across Two Continents. Open Journal of Obstetrics and Gynecology, 5(13),pp.754. (2015).

[8] Jennett P., Hall LA., Hailey D., Ohinmaa A., Anderson C., Thomas R., et al. The socio-economic impact of telehealth: a systematic review. Journal of Telemedicine and Telecare, 9(6),pp.311-20. (2003).

[9] May CR., Finch TL., Cornford J., Exley C., Gately C., Kirk S., et al. Integrating telecare for chronic disease management in the community: what needs to be done? BMC Health Services Research, 11(1),pp.1. (2011).

[10] Ataç A., Kurt E., Yurdakul SE. An overview to ethical problems in telemedicine technology. Procedia-Social and Behavioral Sciences, 103,pp.116-21. (2013).

[11] Hueppmeier RJ., Single A., Welte I. Situation, motivation, implementation and results of telehealth-enabled health care. Journal of Telemedicine and Telecare, 16(4),pp.187-9. (2010).

[12] Ertek S. Tele-medicine and tele-health use in endocrinology practice. Journal of the Acibadem University of Health Science, 2(3),pp.126-30. (2011).

[13] Chi N-C., Demiris G. A systematic review of telehealth tools and interventions to support family caregivers. Journal of Telemedicine and Telecare, 21(1),pp.37-44. (2015).

[14] Louis AA., Turner T., Gretton M., Baksh A., Cleland JG. A systematic review of telemonitoring for the management of heart failure. European Journal of Heart Failure, 5(5),pp.583-90. (2003).

[15] Yuen HK. Effect of a home telecare program on oral health among adults with tetraplegia: a pilot study. Spinal Cord, 51(6):,pp.477-81. (2013).

[16] Huang Z., Tao H., Meng Q., Jing L. Manegement of Endocrine Disease: Effects of telecare intervention on glycemic control in type 2 diabetes: a systematic review and meta-analysis of randomized controlled trials. European Journal of Endocrinology, 172(3),pp.93-101. (2015).

[17] Nocella JM., Dickson VV., Cleland CM., Melkus GDE. Structure, process, and outcomes of care in a telemonitoring program for patients with type 2 diabetes. Patient Related Outcome Measures, 7,pp.19. (2016). 
[18] Davis RM., Hitch AD., Salaam MM., Herman WH., Zimmer-Galler IE., Mayer-Davis EJ. TeleHealth improves diabetes self-management in an underserved community. Diabetes Care, 33(8),pp.1712-7. (2010).

[19] Pérez-Ferre N., Galindo M., Fernández MD., Velasco V., Runkle I., de la Cruz MJ., et al. The outcomes of gestational diabetes mellitus after a telecare approach are not inferior to traditional outpatient clinic visits. International journal of endocrinology. (2010).

[20] Sezgin H., Çınar S. Tip 2 diyabetli hastaların cep telefonu ile takibi: randomize kontrollü çalışma (Follow-up of patients with type 2 diabetes via cell phone: randomized controlled trial). Clinical Experimental Health Science, 3(4),pp.173-183. (2013).

[21] Chen W-L., Chiu W-T., Wu M-S., Hsu M-H., Tsai S-H. Translational research of telecare for the treatment of hepatitis C. BioMed Research International. (2014).

[22] Labbadia LL., D'Innocenzo M., Fogliano RRF., Silva GEF., Queiroz RMR., Carmagnani MIS., et al. Computerized system for managing nursing care indicators at Hospital São Paulo. Revista da Escola de Enfermagem da USP, 45(4),pp.1013-7. (2011).

[23] Acar BG., Pınar G. Telemedicine Practice and Reflections on Nursing. Y1ldırım Beyazit University Journal of Nursing, 1(1),pp.47-54. (2013).

[24] Ersoy S., Yıldırım Y., Şenuzun Aykar F., Fadıloğlu Ç. Innovative field in nursing:Telenursing and telehealth in home care. Journel of Acibadem, 6(4),pp.194-201. (2015).

[25] American Telemedicine Association. Telehealth Nursing Fact Sheet: ATA Telehealth Nursing SIG.[fact sheet]. (2011).

[26] American Telemedicine Association. Telehealth Nursing Fact Sheet: ATA Telehealth Nursing SIG.[fact sheet]. (2011).

[27] Mortensen RA. (Edt). ICNP and Telematic Applications for Nurses in Europe: The telenurse experience. Mantas J In: Integrating Telematics in nursing Education Amsterdam; IOS Press, pp.263-4. (1999).

[28] Erdemir F., Akman A. Telenursing. TURKMIA'09 Proceedings VI National Congress of Medical Informatics, V(1),pp.367-8. (2009).

[29] Nursing. AAoAC. Telehealth Nursing Practice Administration and Practice Standards. Pitman, NJ: American Academy of Ambulatory Care Nursing. 2004. (2004).

[30] Hintistan S., Çilingir D. A Current Approach in Nursing Practice: Telephone Usage. Journal of Nursing Education and Research, 9(1),pp.30-5. (2012).

[31] Yoo SJC., Nyman JA., Cheville AL., Kroenke K. Cost effectiveness of telecare management for pain and depression in patients with cancer: results from a randomized trial. General Hospital Psychiatry, 36(6),pp.599-606. (2014).

[32] Uslu E., Buldukoglu K. The Effect of Tele-Nursing Practices to the Schizophrenia Patient Care: A Systematic Review. Turkish Journal of Psychiatry, 26,pp.1-10. (2015).

[33] Uysal H., Özcan Ş. The effect of individual training and counselling programme for patients with myocardial infarction over patients' quality of life. International Journal of Nursing Practice, 18,pp.445-53. (2012).

[34] American Telemedicine Association. Telehealth nursing fact sheet. (2011).

[35] American Nursing Association. Nursing: Scope and standards of practice: Nursesbooks. org; (2010). 